\title{
Optimal Sobolev imbedding spaces
}

\author{
by \\ Ron Kerman (St. Catharines) and Luboš PICK (Praha)
}

\begin{abstract}
This paper continues our study of Sobolev-type imbedding inequalities involving rearrangement-invariant Banach function norms. In it we characterize when the norms considered are optimal. Explicit expressions are given for the optimal partners corresponding to a given domain or range norm.
\end{abstract}

1. Introduction. Our aim is to further study those rearrangementinvariant Banach function spaces which are optimal in the Sobolev imbeddings considered in [2] and [4].

We begin by briefly describing the content of [4]. Suppose $\Omega$ is a bounded domain in $\mathbb{R}^{n}, n \geq 2$. Let $\partial^{\alpha} / \partial x^{\alpha}:=\partial^{\alpha_{1}+\cdots+\alpha_{n}} / \partial x_{1}^{\alpha_{1}} \ldots \partial x_{n}^{\alpha_{n}}$ be a differential operator of order $|\alpha|:=\alpha_{1}+\cdots+\alpha_{n}$, where $\alpha_{i} \in \mathbb{Z}_{+} \cup\{0\}, i=1, \ldots, n$. Denote by $\left|D^{m} u\right|$ the Euclidean length of the vector, $D^{m} u, 1 \leq m \leq n-1$, of all derivatives of $u$ of order $m$ or less, whenever such derivatives exist on $\Omega$ in the weak sense. In [4] we considered Sobolev imbedding inequalities of the form

$$
\sigma(u) \leq C \varrho\left(\left|D^{m} u\right|\right),
$$

in which $\varrho$ and $\sigma$ are rearrangement-invariant (r.i.) norms (such as those of Lebesgue, Orlicz and Lorentz) and $u$ belongs to the r.i. Sobolev space

$$
W^{m, \varrho}(\Omega):=\left\{u: \Omega \rightarrow \mathbb{R}: \varrho\left(\left|D^{m} u\right|\right)<\infty\right\} ;
$$

that is, we investigated when

$$
W^{m, \varrho}(\Omega) \hookrightarrow L_{\sigma}(\Omega):=\{f: \Omega \rightarrow \mathbb{R}: \sigma(f)<\infty\} .
$$

The focus was on cases in which $\varrho$ and/or $\sigma$ is optimal, namely $W^{m, \varrho}(\Omega)$ cannot be made larger and/or $L_{\sigma}(\Omega)$ cannot be made smaller. Expressions were given for the optimal partners of $\varrho$ and $\sigma$ in (1.1). They involved related r.i. norms, $\bar{\varrho}$ and $\bar{\sigma}$, defined at functions on $I_{\Omega}:=(0,|\Omega|)$. Thus, for $\sigma$, the

2000 Mathematics Subject Classification: Primary 46E35; Secondary 46E30.

Key words and phrases: Sobolev imbeddings, rearrangement-invariant norms, optimality, Hardy operators, supremum operators. 
optimal $\varrho$, called $\varrho_{\sigma}$, had

$$
\varrho_{\sigma}(f):=\sup \bar{\sigma}\left(\int_{t}^{|\Omega|} h(s) s^{m / n-1} d s\right), \quad f: \Omega \rightarrow \mathbb{R},
$$

the supremum being over all $h$ on $I_{\Omega}$ such that

$$
\left|\left\{t \in \mathbb{R}_{+}:|h(t)|>\lambda\right\}\right|=|\{x \in \Omega:|f(x)|>\lambda\}|, \quad \lambda \in \mathbb{R}_{+} ;
$$

as usual, $\mathbb{R}_{+}:=(0, \infty)$. Again, for $\varrho$, the optimal $\sigma$, denoted by $\sigma_{\varrho}$, satisfied

$$
\sigma_{\varrho}^{\prime}(g):=\bar{\varrho}^{\prime}\left(t^{m / n-1} \int_{0}^{t} g^{*}(s) d s\right), \quad g: \Omega \rightarrow \mathbb{R},
$$

where $\sigma_{\varrho}^{\prime}$ and $\bar{\varrho}^{\prime}$ are the Köthe dual norms of $\sigma_{\varrho}$ and $\bar{\varrho}$ discussed in Section 2 below and

$$
g^{*}(t):=\inf \left\{\lambda>0: \mu_{g}(\lambda) \leq t\right\}, \quad t \in I_{\Omega},
$$

with

$$
\mu_{g}(\lambda):=|\{x \in \Omega:|g(x)|>\lambda\}|, \quad \lambda \in \mathbb{R}_{+},
$$

is the decreasing rearrangement of $g$ on $I_{\Omega}$.

Proposition 5.2 in [4] proved that the formula for $\varrho_{\sigma}$ can be dramatically improved if $\sigma$ is optimal in (1.1). There is also a more explicit formula for $\sigma_{\varrho}$ when $\varrho$ is optimal in (1.1). These expressions, together with precise criteria for the optimality of $\varrho$ and $\sigma$ in (1.1), are the subject of Theorem A below.

To state the theorem we must, first of all, introduce two supremum operators, namely,

and

$$
\left(S_{n / m} f\right)(t):=t^{m / n-1} \sup _{0<s \leq t} s^{1-m / n} f^{*}(s)
$$

$$
\left(T_{n / m} f\right)(t):=t^{-m / n} \sup _{t \leq s<|\Omega|} s^{m / n} f^{*}(s), \quad f: I_{\Omega} \rightarrow \mathbb{R}, t \in I_{\Omega} .
$$

Observe that for $S_{n / m} f$ to be finite one requires

$$
\sup _{0<s \leq|\Omega|} s^{1-m / n} f^{*}(s)<\infty,
$$

or, as we will write, $f \in L_{n /(n-m), \infty}\left(I_{\Omega}\right)$. Also, one has

$$
\left(S_{n / m} f\right)^{* *}(t) \approx\left(S_{n / m} f^{* *}\right)(t) \approx\left(S_{n / m} f\right)(t), \quad f \in \mathfrak{M}_{+}\left(I_{\Omega}\right), t \in I_{\Omega} .
$$

(We recall the notation $X \approx Y$, which signifies that each of $X$ and $Y$ is dominated by a constant multiple of the other, the constants being independent of all functions involved. More generally, $X \lesssim Y$ means $X$ is no bigger than a constant times $Y$, with the constant independent of all functions involved.) For any measurable subset $E$ of $\mathbb{R}^{n}$, we define

$$
\mathfrak{M}(E):=\{f: E \rightarrow \mathbb{R}: f \text { is measurable }\}
$$

and denote by $\mathfrak{M}_{+}(E)$ the class of nonnegative functions in $\mathfrak{M}(E)$. 
Theorem A. Fix $m, n \in \mathbb{Z}_{+}$, with $n \geq 2$ and $1 \leq m \leq n-1$. Let $\Omega$ be a bounded Lipschitz domain in $\mathbb{R}^{n}$. Then, an r.i. norm $\varrho$ on $\mathfrak{M}_{+}(\Omega)$, associated to the r.i. norm $\bar{\varrho}$ on $\mathfrak{M}_{+}\left(I_{\Omega}\right)$, with $L_{\bar{\varrho}}\left(I_{\Omega}\right) \supsetneq L_{n / m, 1}\left(I_{\Omega}\right)$, is optimal in (1.1) for some r.i. norm $\sigma$ on $\mathfrak{M}_{+}(\Omega)$ if and only if

$$
S_{n / m}: L_{\bar{\varrho}^{\prime}}\left(I_{\Omega}\right) \rightarrow L_{\bar{\varrho}^{\prime}}\left(I_{\Omega}\right) .
$$

In that case,

$$
\sigma_{\varrho}(f) \approx \bar{\varrho}\left(t^{-m / n}\left[f^{* *}(t)-f^{*}(t)\right]\right)+\int_{0}^{1} f^{*}(t) d t, \quad f \in \mathfrak{M}_{+}(\Omega),
$$

where $f^{* *}(t):=t^{-1} \int_{0}^{t} f^{*}(s) d s$.

Again, an r.i. norm $\sigma$ on $\mathfrak{M}_{+}(\Omega)$, associated to the r.i. norm $\bar{\sigma}$ on $\mathfrak{M}_{+}\left(I_{\Omega}\right)$, is optimal in (1.1) for some r.i. norm $\varrho$ on $\mathfrak{M}_{+}(\Omega)$ if and only if

$$
T_{n / m}: L_{\bar{\sigma}^{\prime}}\left(I_{\Omega}\right) \rightarrow L_{\bar{\sigma}^{\prime}}\left(I_{\Omega}\right),
$$

in which case

$$
\varrho_{\sigma}(f) \approx \bar{\sigma}\left(\int_{t}^{|\Omega|} f^{*}(s) s^{m / n-1} d s\right), \quad f \in \mathfrak{M}_{+}(\Omega) .
$$

In practice, one starts with a Sobolev space, $W^{m, \varrho}(\Omega)$, and seeks to find its optimal imbedding space, $L_{\sigma_{\varrho}}(\Omega)$. One can then go on to form $\varrho_{D}:=\varrho_{\sigma_{\varrho}}$. It is readily seen that

$$
W^{m, \varrho}(\Omega) \hookrightarrow W^{m, \varrho_{D}}(\Omega) \hookrightarrow L_{\sigma_{\varrho}}(\Omega)
$$

and, indeed, that $W^{m, \varrho_{D}}(\Omega)$ is the largest Sobolev space that imbeds into $L_{\sigma_{\varrho}}(\Omega)$. Accordingly, we refer to $\varrho_{D}$ as the optimal r.i. hull norm for $\varrho$ in (1.1). Our new description of $\varrho_{D}$ is given in

Theorem B. Fix $m, n \in \mathbb{Z}_{+}$, with $n \geq 2$ and $1 \leq m \leq n-1$. Let $\Omega$ be a bounded Lipschitz domain in $\mathbb{R}^{n}$ and suppose $\varrho$ is an r.i. norm on $\mathfrak{M}_{+}(\Omega)$, associated to the r.i. norm $\bar{\varrho}$ on $\mathfrak{M}_{+}\left(I_{\Omega}\right)$. Then,

$$
\varrho_{D}(f) \approx \mu^{\prime}\left(f^{*}\right), \quad f \in \mathfrak{M}_{+}(\Omega),
$$

where

$$
\mu(g):=\varrho^{\prime}\left(S_{n / m} g^{* *}\right), \quad g \in \mathfrak{M}_{+}\left(I_{\Omega}\right) .
$$

The basic technical result on which the proofs of Theorems A and B depend is

Proposition C. Fix $m, n \in \mathbb{Z}_{+}$, with $n \geq 2$ and $1 \leq m \leq n-1$. Let $\Omega$ be a bounded Lipschitz domain in $\mathbb{R}^{n}$ and suppose $\varrho$ is an r.i. norm on $\mathfrak{M}_{+}(\Omega)$, associated to the r.i. norm $\bar{\varrho}$ on $\mathfrak{M}_{+}\left(I_{\Omega}\right)$ satisfying $L_{\bar{\varrho}}\left(I_{\Omega}\right) \supsetneq L_{n / m, 1}\left(I_{\Omega}\right)$. 
Then,

$$
\sigma_{\varrho}(f) \approx \sup _{\varrho^{\prime}\left(S_{n / m} g\right) \leq 1} \int_{0}^{|\Omega|} t^{-m / n}\left[f^{* *}(t)-f^{*}(t)\right] g^{*}(t) d t+\int_{0}^{|\Omega|} f^{*}(t) d t,
$$

where $f \in \mathfrak{M}_{+}(\Omega), g \in \mathfrak{M}_{+}\left(I_{\Omega}\right)$.

The structure of the paper is as follows. Section 2 contains background material on r.i. norms and an interpolation-theoretic result involving $S_{n / m}$ and $T_{n / m}$ needed later on. The optimal range, $\sigma_{\varrho}$, corresponding to a given $\varrho$, is treated in Section 3, which begins with the proof of Proposition C. Theorems $\mathrm{A}$ and $\mathrm{B}$ are proved in Section 4.

Theorem A is illustrated in the context of Orlicz spaces in the last section, using results from [3]. A property of the so-called level function, $f^{\circ}$, of $f \in \mathfrak{M}\left(I_{\Omega}\right)$, necessary to obtain (1.4), is proved in an appendix.

Finally, we mention that, in [5], Proposition $\mathrm{C}$ turns out to be crucial to characterizing when the imbedding

$$
W^{m, \varrho}(\Omega) \hookrightarrow L_{\sigma}(\Omega)
$$

is compact.

2. Rearrangement-invariant norms. The decreasing rearrangement defined above satisfies [1, Chapter 2, Theorem 2.2]

$$
\int_{\Omega} f(x) g(x) d x \leq \int_{0}^{|\Omega|} f^{*}(t) g^{*}(t) d t, \quad f, g \in \mathfrak{M}_{+}(\Omega) .
$$

The operation of rearrangement is not sublinear, though for the Hardy average of $h^{*}$, namely $h^{* *}(t):=t^{-1} \int_{0}^{t} h^{*}(s) d s, t \in I_{\Omega}$, we have [1, Chapter 2, Proposition 3.3]

$$
(f+g)^{* *}(t) \leq f^{* *}(t)+g^{* *}(t), \quad f, g \in \mathfrak{M}_{+}(\Omega), t \in I_{\Omega} .
$$

DEFINITION 2.1. A rearrangement-invariant (r.i.) Banach function norm $\varrho$ on $\mathfrak{M}_{+}(\Omega)$ satisfies the following seven axioms:

$\left(A_{1}\right) \varrho(f) \geq 0$, with $\varrho(f)=0$ if and only if $f=0$ a.e. on $\Omega$;

$\left(A_{2}\right) \varrho(c f)=c \varrho(f), c \geq 0$

$\left(A_{3}\right) \varrho(f+g) \leq \varrho(f)+\varrho(g)$;

$\left(A_{4}\right) f_{n} \uparrow f$ implies $\varrho\left(f_{n}\right) \uparrow \varrho(f)$;

$\left(A_{5}\right) \varrho\left(\chi_{E}\right)<\infty$ for measurable $E \subset \Omega,|E|<\infty$;

$\left(A_{6}\right) \int_{E} f(x) d x \leq C_{E} \varrho(f)$, with $E \subset \Omega,|E|<\infty, C_{E}>0$ independent of $f$;

$\left(A_{7}\right) \varrho(f)=\varrho(g)$ whenever $\mu_{f}=\mu_{g}$.

According to a fundamental result of Luxemburg [1, Chapter 2, Theorem 4.10], to every r.i. norm $\varrho$ on $\mathfrak{M}_{+}(\Omega)$ there corresponds an r.i. norm, $\bar{\varrho}$, 
on $\mathfrak{M}_{+}\left(I_{\Omega}\right)$, such that

$$
\varrho(f)=\bar{\varrho}\left(f^{*}\right), \quad f \in \mathfrak{M}_{+}(\Omega) .
$$

The basic technique for working with an r.i. norm $\varrho$ involves the HardyLittlewood-Pólya (HLP) Principle (see [1, Chapter 2, Proposition 4.6]), which asserts that

$$
f^{* *}(t) \leq g^{* *}(t), t \in I_{\Omega}, \quad \text { implies } \varrho(f) \leq \varrho(g) .
$$

It is based on the following result of Hardy: if $f, g, h \in \mathfrak{M}_{+}\left(I_{\Omega}\right)$, then

$$
\begin{aligned}
\int_{0}^{t} f(s) d s \leq \int_{0}^{t} g(s) d s, \quad t \in I_{\Omega} & \\
& \Rightarrow \int_{0}^{|\Omega|} f(t) h^{*}(t) d t \leq \int_{0}^{|\Omega|} g(t) h^{*}(t) d t .
\end{aligned}
$$

The Köthe dual of an r.i. norm $\varrho$ on $\mathfrak{M}_{+}(\Omega)$ is another such norm, $\varrho^{\prime}$, with

$$
\varrho^{\prime}(g):=\sup _{\varrho(h) \leq 1} \int_{\Omega} g(x) h(x) d x, \quad g, h \in \mathfrak{M}_{+}(\Omega) .
$$

It obeys the Principle of Duality,

$$
\varrho^{\prime \prime}:=\left(\varrho^{\prime}\right)^{\prime}=\varrho .
$$

Further, the Hölder inequality,

$$
\int_{\Omega} f(x) g(x) d x \leq \varrho(f) \varrho^{\prime}(g),
$$

holds for all $f, g \in \mathfrak{M}_{+}(\Omega)$, and this inequality is saturated, in the sense that, given $f \in \mathfrak{M}_{+}(\Omega)$ and $\varepsilon>0$, there exists $g_{0} \in \mathfrak{M}_{+}(\Omega)$ such that $\varrho^{\prime}\left(g_{0}\right)=1$ and

$$
\int_{\Omega} f(x) g_{0}(x) d x>(1-\varepsilon) \varrho(f) .
$$

Finally, $\overline{\varrho^{\prime}}=\bar{\varrho}^{\prime}$.

A smaller functional dual to the r.i. norm $\bar{\varrho}$ on $\mathfrak{M}\left(I_{\Omega}\right)$ will also be of interest to us, namely the down dual norm, $\bar{\varrho}_{d}^{\prime}$, defined by

$$
\bar{\varrho}_{d}^{\prime}(g):=\sup _{\bar{\varrho}(h) \leq 1} \int_{0}^{|\Omega|} g(t) h^{*}(t) d t, \quad g, h \in \mathfrak{M}_{+}\left(I_{\Omega}\right) .
$$

One connection between $\varrho^{\prime}$ and $\varrho_{d}^{\prime}$, observed in [2, p. 312], is

$$
\varrho^{\prime}(g)=\bar{\varrho}_{d}^{\prime}\left(g^{*}\right), \quad g \in \mathfrak{M}_{+}\left(I_{\Omega}\right) .
$$

Recently, G. Sinnamon [7] proved

$$
\bar{\varrho}_{d}^{\prime}(g)=\bar{\varrho}^{\prime}\left(g^{\circ}\right), \quad g \in \mathfrak{M}_{+}\left(I_{\Omega}\right),
$$


in which $g^{\circ}$, referred to as the level function of $g$, is the (nonincreasing) derivative of the least concave majorant of $\int_{0}^{t} g(s) d s, t \in I_{\Omega}$. One has

$$
\int_{0}^{t} g^{*}(s) d s \geq \int_{0}^{t} g^{\circ}(s) d s \approx t \sup _{t \leq s<|\Omega|} s^{-1} \int_{0}^{s} g(y) d y, \quad g \in \mathfrak{M}_{+}\left(I_{\Omega}\right) .
$$

The inequality in (2.7) is almost obvious. The equivalence was pointed out to us by G. Sinnamon ([8]); a proof of it, due to A. Gogatishvili, appears in the appendix at the end of this paper.

Corresponding to an r.i. norm $\varrho$ on $\mathfrak{M}_{+}(\Omega)$ is the set

$$
L_{\varrho}(\Omega):=\{f \in \mathfrak{M}(\Omega): \varrho(|f|)<\infty\},
$$

which becomes a Banach space when

$$
\|f\|_{L_{\varrho}(\Omega)}:=\varrho(|f|), \quad f \in L_{\varrho}(\Omega) ;
$$

indeed, it is a so-called rearrangement-invariant Banach function space, or, for short, an r.i. space. A detailed treatment of such spaces appears in [1, Chapters 1 and 2].

The dilation operator $E_{s}, s \in \mathbb{R}_{+}$, given at $f \in \mathfrak{M}_{+}\left(I_{\Omega}\right), t \in I_{\Omega}$, by

$$
\left(E_{s} f\right)(t):= \begin{cases}f(t / s), & 0<t \leq|\Omega| s, \\ 0, & |\Omega| s<t<|\Omega|,\end{cases}
$$

if $s \in(0,1)$, and by

$$
\left(E_{s} f\right)(t):=f(t / s), \quad 0<t \leq|\Omega|,
$$

if $s \in[1, \infty)$, is bounded on any r.i. space $L_{\bar{\varrho}}\left(I_{\Omega}\right)$ ([1, Chapter 3, Proposition 5.11]).

The Lorentz norms, $\varrho_{p, q}$, with $1<p<\infty, 1 \leq q \leq \infty$, are defined by

$$
\varrho_{p, q}(f):=\left(\int_{0}^{|\Omega|}\left(f^{* *}(t) t^{1 / p-1 / q}\right)^{q} d t\right)^{1 / q} \quad \text { when } q<\infty,
$$

and

$$
\varrho_{p, \infty}(f):=\sup _{0<t<|\Omega|} t^{1 / p} f^{* *}(t), \quad f \in \mathfrak{M}_{+}(\Omega) .
$$

In view of a well-known inequality of Hardy,

$$
\varrho_{p, p}(f) \approx\|f\|_{p}:=\left(\int_{\Omega} f(x)^{p} d x\right)^{1 / p}=\left(\int_{0}^{|\Omega|} f^{*}(t)^{p} d t\right)^{1 / p}, \quad f \in \mathfrak{M}_{+}(\Omega) .
$$

We denote $L_{\varrho_{p, q}}(\Omega)$ by $L_{p, q}(\Omega)$.

To conclude, we record a special interpolation-theoretic result.

Suppose $X_{0}, X_{1}$ and $X$ are r.i. spaces of functions in $\mathfrak{M}_{+}(\Omega)$ satisfying

$$
X_{0} \subset X \subset X_{1} \quad \text { or } \quad X_{0} \supset X \supset X_{1} \text {. }
$$


We say that $X$ is an interpolation space between $X_{0}$ and $X_{1}$, denoted $X \in$ $\operatorname{Int}\left(X_{0}, X_{1}\right)$, if, for any linear operator $T$,

$$
T: X_{0} \rightarrow X_{0} \text { and } T: X_{1} \rightarrow X_{1} \text { implies } T: X \rightarrow X .
$$

For example, if $\varrho$ is any r.i. norm on $\mathfrak{M}_{+}(\Omega)$, then

$$
L_{1}(\Omega) \supset L_{\varrho}(\Omega) \supset L_{\infty}(\Omega) \quad \text { and } \quad L_{\varrho}(\Omega) \in \operatorname{Int}\left(L_{1}(\Omega), L_{\infty}(\Omega)\right) ;
$$

see [1, Chapter 3, Theorem 2.12].

When $X_{0}$ and $X_{1}$ are certain Lorentz spaces, there are simple tests for $L_{\varrho}(\Omega) \in \operatorname{Int}\left(X_{0}, X_{1}\right)$ involving the supremum operators $S_{n / m}$ and $T_{n / m}$. More specifically, we have

TheOREM 2.2. Let $m, n \in \mathbb{Z}_{+}$with $n \geq 2$ and $1 \leq m \leq n-1$, and suppose $\Omega$ is a bounded Lipschitz domain in $\mathbb{R}^{n}$. Let $\varrho$ be an r.i. norm on $\mathfrak{M}_{+}(\Omega)$. Then $L_{\varrho}(\Omega) \supset L_{n / m, 1}(\Omega)$, and

$$
L_{\varrho}(\Omega) \in \operatorname{Int}\left(L_{1}(\Omega), L_{n / m, 1}(\Omega)\right)
$$

if and only if (1.3) holds.

Again, given $L_{\varrho}(\Omega) \subset L_{n /(n-m), 1}(\Omega)$, we have

$$
L_{\varrho}(\Omega) \in \operatorname{Int}\left(L_{n /(n-m), 1}(\Omega), L_{\infty}(\Omega)\right)
$$

if and only if (1.5) holds.

The "if" parts are consequences of [4, Corollary 3.7 and Theorem 3.12]. The "only if" parts follow by standard arguments (see, for example, [1, Chapter 4, Section 4]) from the endpoint estimates for $S_{n / m}$ and $T_{n / m}$, in [4, Lemma 3.5], combined with their "quasisubadditivity" properties

$$
\left(S_{n / m}[f+g]\right)(t) \leq\left(S_{n / m} f\right)(t / 2)+\left(S_{n / m} g\right)(t / 2)
$$

and

$$
\left(T_{n / m}[f+g]\right)(t) \leq\left(T_{n / m} f\right)(t / 2)+\left(T_{n / m} g\right)(t / 2), \quad f, g \in \mathfrak{M}_{+}\left(I_{\Omega}\right), t \in I_{\Omega},
$$

and the boundedness of the dilation operators on every r.i. space.

One readily sees from $[4$, Theorem $\mathrm{A}]$ that

$$
\sigma_{\varrho_{1}}=\varrho_{n /(n-m), 1} \text { and } \varrho_{\varrho_{\infty}}=\varrho_{n / m, 1} \text {. }
$$

Thus, when considering $\varrho$ and $\sigma$ in (1.1) one may safely assume

$$
L_{\varrho}(\Omega) \supset L_{n / m, 1}(\Omega) \text { and } L_{\sigma}(\Omega) \subset L_{n /(n-m), 1}(\Omega) .
$$

3. The optimal range norm $\sigma_{\varrho}$. In the first part of this section we prove Proposition C. The strategy of the proof is as follows. According to $(1.2)$,

$$
\sigma_{\varrho}^{\prime}(g)=\bar{\varrho}^{\prime}\left(t^{m / n-1} \int_{0}^{t} g^{*}(s) d s\right)=: \lambda(g), \quad g \in \mathfrak{M}_{+}(\Omega) .
$$

Thus, we must show $\lambda^{\prime}(f)$ is equivalent to the right side of (1.7). 
We begin with two lemmas essential to the proof.

Lemma 3.1. Fix $b>0$ and set $I_{b}:=(0, b)$. Let $\bar{\varrho}$ be an r.i. norm on $\mathfrak{M}_{+}\left(I_{b}\right)$ such that $L_{\bar{\varrho}^{\prime}}\left(I_{b}\right) \subsetneq L_{n /(n-m), \infty}\left(I_{b}\right)$. Then,

$$
\begin{aligned}
\mu(f):=\sup _{\bar{\varrho}^{\prime}\left(S_{n / m} g\right) \leq 1} \int_{0}^{b} f^{*}(t) d \operatorname{csup}_{0<s \leq t} s^{1-m / n} g^{*}(s)+\int_{0}^{|\Omega|} f^{*}(s) d s, \\
\quad f, g \in \mathfrak{M}_{+}\left(I_{b}\right),
\end{aligned}
$$

is also an r.i. norm on $\mathfrak{M}_{+}\left(I_{b}\right)$; in (3.2), $\operatorname{csup}_{0<s \leq t} s^{1-m / n} g^{*}(s)=: \alpha(t)$ denotes the least concave majorant of $\sup _{0<s \leq t} s^{1-m / n} g^{*}(s)=: \beta(t), t \in I_{b}$, and

$$
\int_{0}^{b} f^{*}(t) d \alpha(t):=\lim _{\varepsilon \rightarrow 0+} \int_{\varepsilon}^{b-\varepsilon} f^{*}(t) d \alpha(t) .
$$

Proof. To start, observe that $\beta(t)$ is quasiconcave (so $\beta(t) \leq \alpha(t) \leq$ $2 \beta(t))$ and that $\bar{\varrho}^{\prime}\left(S_{n / m} g\right)<\infty$ implies $\beta(b-)=\left(S_{n / m} g\right)(b-)<\infty$. Thus, $\alpha(t)$ is continuous on $I_{b}$ (in fact, locally Lipschitz of order 1 ) and hence

$$
\int_{\varepsilon}^{1-\varepsilon} f^{*}(t) d \alpha(t)
$$

is well defined as a (Riemann) Stieltjes integral, for all $\varepsilon$ with $0<\varepsilon<b / 2$. Indeed,

$$
\int_{\varepsilon}^{b-\varepsilon} f^{*}(t) d \alpha(t)=\int_{\varepsilon}^{b-\varepsilon} f^{*}(t) h(t) d t,
$$

hence

$$
\int_{0}^{b} f^{*}(t) d \alpha(t)=\int_{0}^{b} f^{*}(t) h(t) d t,
$$

where $h(t):=d \alpha(t) / d t$ is nonincreasing.

As for $\mu$ being an r.i. norm, only the subadditivity requires comment. But, it readily follows once we observe that, given $f_{1}, f_{2} \in \mathfrak{M}_{+}\left(I_{b}\right),(2.2)$ and (2.4) ensure

$$
\begin{aligned}
\int_{0}^{b}\left(f_{1}+f_{2}\right)^{*}(t) d \alpha(t) & =\int_{0}^{b}\left(f_{1}+f_{2}\right)^{*}(t) h(t) d t \leq \int_{0}^{b}\left[f_{1}^{*}(t)+f_{2}^{*}(t)\right] h(t) d t \\
& =\int_{0}^{b} f_{1}^{*}(t) d \alpha(t)+\int_{0}^{b} f_{2}^{*}(t) d \alpha(t) . \text { - }
\end{aligned}
$$

Lemma 3.2. Suppose $\varrho$ is an r.i. norm on $\mathfrak{M}_{+}(\Omega)$, associated to the r.i. norm $\bar{\varrho}$ on $\mathfrak{M}_{+}\left(I_{\Omega}\right)$, with $L_{\bar{\varrho}}\left(I_{\Omega}\right) \subsetneq L_{n / m, \infty}\left(I_{\Omega}\right)$, and let $\lambda$ be defined as 
in (3.1). Then, $\lambda^{\prime} \approx \tau$ with

$$
\tau(f):=\sup _{\bar{\varrho}^{\prime}\left(S_{n / m} g\right) \leq 1} \int_{0}^{|\Omega|}-t^{1-m / n} g^{*}(t) d f^{*}(t)+\int_{0}^{|\Omega|} f^{*}(t) d t
$$

for $f \in \mathfrak{M}(\Omega), g \in C\left(I_{\Omega}\right)$.

Proof. In view of Corollary 3.7 and Theorem 3.13 of [4], we may assume

$$
\lambda(g) \approx \nu\left(t^{m / n-1} \int_{0}^{t} g^{*}(s) d s\right), \quad g \in \mathfrak{M}(\Omega),
$$

where

$$
\nu(h)=\bar{\varrho}^{\prime}\left(S_{n / m} h^{* *}\right) \approx \varrho^{\prime}\left(S_{n / m} h\right), \quad h \in \mathfrak{M}\left(I_{\Omega}\right),
$$

and

$$
S_{n / m}: L_{\nu}\left(I_{\Omega}\right) \rightarrow L_{\nu}\left(I_{\Omega}\right) .
$$

We first show that $\tau^{\prime} \lesssim \lambda$. For any $f, g \in C\left(I_{\Omega}\right)$, with $f^{*}(0+)<\infty$ and $f^{*}(|\Omega|-)=0$, we have

$$
\begin{aligned}
& \int_{0}^{|\Omega|} g^{*}(t) f^{*}(t) d t \leq \int_{0}^{|\Omega|} g^{*}(t) \int_{t}^{|\Omega|}-d f^{*}(s) d t=\int_{0}^{|\Omega|}-\int_{0}^{t} g^{*}(s) d s d f^{*}(t) \\
& =\int_{0}^{|\Omega|}-t^{1-m / n} t^{m / n-1} \int_{0}^{t} g^{*}(s) d s d f^{*}(t) \\
& \leq \int_{0}^{|\Omega|}-t^{1-m / n} \sup _{t \leq s<|\Omega|} s^{m / n-1} \int_{0}^{s} g^{*}(y) d y d f^{*}(t) \\
& \lesssim \lambda(g) \nu\left(t^{m / n-1} \int_{0}^{t} g^{*}(s) d s\right)^{-1} \int_{0}^{|\Omega|}-t^{1-m / n} \sup _{t \leq s<|\Omega|} s^{m / n-1} \int_{0}^{s} g^{*}(y) d y d f^{*}(t) \\
& \lesssim \lambda(g) \nu\left(\sup _{t \leq s<|\Omega|} s^{m / n-1} \int_{0}^{s} g^{*}(y) d y\right)^{-1} \\
& \times \int_{0}^{|\Omega|}-t^{1-m / n} \sup _{t \leq s<|\Omega|} s^{m / n-1} \int_{0}^{s} g^{*}(y) d y d f^{*}(t) \\
& |\Omega| \\
& \lesssim \lambda(g) \sup _{\nu(h) \leq 1} \int_{0}-t^{1-m / n} h^{*}(t) d f^{*}(t) \lesssim \lambda(g) \tau(f),
\end{aligned}
$$

in which (3.3), Theorem 3.9 of [4] and (3.4) combined with (3.5) were used to obtain the fourth last, third last and second last inequalities, respectively. Thus, $\tau^{\prime} \lesssim \lambda$. 
To prove $\lambda \lesssim \tau^{\prime}$ we show the existence of $C>0$ such that to each $g \in$ $\mathfrak{M}_{+}(\Omega), \lambda(g)<\infty$, there corresponds $f_{0} \in \mathfrak{M}_{+}(\Omega)$ satisfying $f_{0}^{*}(0+)<\infty$, $f_{0}^{*}(|\Omega|-)=0, \tau\left(f_{0}\right) \leq C$ and

$$
\int_{0}^{|\Omega|} g^{*}(t) f_{0}^{*}(t) d t \geq C^{-1} \lambda(g) .
$$

Now, $\lambda(g)<\infty$ implies the existence of $k_{0} \in \mathfrak{M}_{+}\left(I_{\Omega}\right)$, with $\varrho\left(k_{0}\right) \leq 1$, such that

$$
\int_{0}^{|\Omega|} k_{0}(t) t^{m / n-1} \int_{0}^{t} g^{*}(s) d s d t>\frac{1}{2} \lambda(g) .
$$

Take $f_{0}$ such that

$$
f_{0}^{*}(t)=\int_{t}^{|\Omega|} k_{0}(s) s^{m / n-1} d s, \quad t \in I_{\Omega} .
$$

Then, for $h=h^{*} \in \mathfrak{M}_{+}\left(I_{\Omega}\right)$ with $\nu(h) \leq 1$,

$$
\begin{aligned}
\int_{0}^{|\Omega|}-t^{1-m / n} h^{*}(t) d f_{0}(t) & =\int_{0}^{|\Omega|} h^{*}(t) k_{0}^{*}(t) d t \leq \nu(h) \nu^{\prime}\left(k_{0}\right) \\
& \lesssim \nu(h) \bar{\varrho}\left(k_{0}\right) \quad\left(\bar{\varrho}^{\prime} \leq \nu \text { implies } \nu^{\prime} \leq \bar{\varrho}\right) \\
& \leq C,
\end{aligned}
$$

and

$$
\begin{aligned}
\int_{0}^{|\Omega|} f_{0}^{*}(t) d t & =\int_{0}^{|\Omega|} \int_{t}^{|\Omega|} k_{0}(s) s^{m / n-1} d s d t=\int_{0}^{|\Omega|} k_{0}(t) t^{m / n} d t \\
& \lesssim \int_{0}^{|\Omega|} k_{0}(t) d t \lesssim \varrho\left(k_{0}\right) \leq C,
\end{aligned}
$$

so $\tau\left(f_{0}\right) \leq C$. Further,

$$
\begin{aligned}
\int_{0}^{|\Omega|} g^{*}(t) f_{0}^{*}(t) d t & =\int_{0}^{|\Omega|} g^{*}(t) \int_{t}^{1} k_{0}(s) s^{m / n-1} d s d t \\
& =\int_{0}^{|\Omega|} k_{0}(t) t^{m / n-1} \int_{0}^{t} g^{*}(s) d s d t \geq \frac{1}{2} \lambda(g) .
\end{aligned}
$$

The result will follow by the Principle of Duality once we verify

$$
\tau(f) \approx \mu(f), \quad f \in \mathfrak{M}_{+}(\Omega), f^{*}(0+)<\infty, f^{*}(|\Omega|-)=0,
$$

where $\mu(f)$ is defined as in (3.2) with $b=|\Omega|$, since $\mu$ was shown to be an r.i. norm in Lemma 3.1. 
When $g=g^{*} \in C\left(I_{\Omega}\right)$ with $g^{*}(0+)<\infty$,

$$
\lim _{t \rightarrow 0+} f^{*}(t) \operatorname{csup}_{0<s \leq t} s^{1-m / n} g^{*}(s)=\lim _{t \rightarrow|\Omega|-} f^{*}(t) \operatorname{csup}_{0<s \leq t} s^{1-m / n} g^{*}(s)=0,
$$

and, thus, integration by parts yields

$$
\begin{aligned}
\int_{0}^{|\Omega|} f^{*}(t) d \operatorname{csup}_{0<s \leq t} s^{1-m / n} g^{*}(s) & =\int_{0}^{|\Omega|}-\operatorname{csup}_{0<s \leq t} s^{1-m / n} g^{*}(s) d f^{*}(t) \\
& \geq \int_{0}^{1}-t^{1-m / n} g^{*}(t) d f^{*}(t),
\end{aligned}
$$

whence

$$
\mu(f) \geq \tau(f), \quad f \in \mathfrak{M}_{+}\left(I_{\Omega}\right)
$$

Again,

$$
\begin{aligned}
\sup _{\bar{\varrho}^{\prime}\left(S_{n / m} g\right) \leq 1} & \int_{0}^{|\Omega|}-\operatorname{csup}_{0<s \leq t} s^{1-m / n} g^{*}(s) d f^{*}(t) \\
& \lesssim \sup _{\nu(g) \leq 1} \int_{0}^{|\Omega|}-t^{1-m / n} t^{m / n-1} \operatorname{csup}_{0<s \leq t} s^{1-m / n} g^{*}(s) d f^{*}(t) \\
& \lesssim \sup _{\nu(g) \leq 1} \int_{0}^{|\Omega|}-t^{1-m / n}\left(S_{n / m} g\right)(t) d(t) \\
& \lesssim \sup _{\nu\left(S_{n / m} g\right) \leq 1} \int_{0}^{|\Omega|}-t^{1-m / n}\left(S_{n / m} g\right)(t) d f^{*}(t) \quad \text { by }(3.5) \\
& \lesssim \sup _{\nu(g) \leq 1} \int_{0}^{|\Omega|}-t^{1-m / n} g^{*}(t) d f^{*}(t) \\
& \lesssim \sup _{\bar{\varrho}^{\prime}\left(S_{n / m} g\right) \leq 1} \int_{0}^{|\Omega|}-t^{1-m / n} g^{*}(t) d f^{*}(t) \quad\left(g=g^{*} \in \mathfrak{M}_{+}\left(I_{\Omega}\right)\right) \\
& \lesssim \tau(f) .
\end{aligned}
$$

To get the second line of the last chain of inequalities, we have used the quasiconcavity of $\beta(t)=\sup _{0<s \leq t} s^{1-m / n} g^{*}(s), t \in I_{\Omega}$.

Proof of Proposition C. In view of Lemma 3.2, $\sigma_{\varrho}$ satisfies

$$
\sigma_{\varrho}(f) \approx \sup _{\varrho^{\prime}\left(S_{n / m} g\right) \leq 1} \int_{0}^{|\Omega|}-t^{1-m / n} g^{*}(t) d f^{*}(t)+\int_{0}^{|\Omega|} f^{*}(t) d t,
$$

where $f \in \mathfrak{M}_{+}(\Omega)$ and $g \in C\left(I_{\Omega}\right)$. Define the operator $P$ by 


$$
(P h)(t):=t^{-1} \int_{0}^{t} h(s) d s, \quad h \in \mathfrak{M}_{+}\left(I_{\Omega}\right), t \in I_{\Omega} .
$$

According to [4, Theorem 3.12], $L_{\sigma_{\varrho}}\left(I_{\Omega}\right)$ is an interpolation space between $L_{n /(n-m), 1}\left(I_{\Omega}\right)$ and $L_{\infty}\left(I_{\Omega}\right)$, hence Theorem 5.15 in Chapter 3 of [1] ensures

$$
P: L_{\sigma_{\varrho}}\left(I_{\Omega}\right) \rightarrow L_{\sigma_{\varrho}}\left(I_{\Omega}\right) \text {. }
$$

This means we can replace $f^{*}(t)$ by $f^{* *}(t)$ and, indeed, by $t^{-1} \int_{0}^{t} f^{* *}(s) d s$, on the right side of (3.6).

Now, for each $\varepsilon$ with $0<\varepsilon<|\Omega| / 2$,

$$
\begin{aligned}
& \int_{\varepsilon}^{|\Omega|-\varepsilon}-t^{1-m / n} g^{*}(t) d\left[t^{-1} \int_{0}^{t} f^{* *}(s) d s\right] \\
&=\int_{\varepsilon}^{|\Omega|-\varepsilon}-t^{1-m / n} g^{*}(t)\left[-t^{-2} \int_{0}^{t} f^{* *}(s) d s+t^{-1} f^{* *}(t)\right] d t \\
&=\int_{\varepsilon}^{|\Omega|-\varepsilon} t^{-m / n}\left[t^{-1} \int_{0}^{t}\left[f^{* *}(s)-f^{*}(s)\right] d s\right] g^{*}(t) d t
\end{aligned}
$$

SO

$$
\begin{aligned}
& \int_{0}^{|\Omega|}-t^{1-m / n} g^{*}(t) d\left[t^{-1} \int_{0}^{t} f^{* *}(s) d s\right] \\
& =\int_{0}^{|\Omega|} t^{-m / n}\left[t^{-1} \int_{0}^{t}\left[f^{* *}(s)-f^{*}(s)\right] d s\right] g^{*}(t) d t \\
& =\int_{0}^{|\Omega|} t^{-m / n}\left[f^{* *}(t)-f^{*}(t)\right]\left[t^{m / n} \int_{t}^{|\Omega|} g^{*}(s) s^{-m / n-1} d s\right] d t .
\end{aligned}
$$

Again, the operator $R_{n / m}$, defined by

$$
\left(R_{n / m} h\right)(t):=t^{m / n} \int_{t}^{|\Omega|} h(s) s^{-m / n-1} d s, \quad h \in \mathfrak{M}_{+}\left(I_{\Omega}\right), t \in I_{\Omega},
$$

satisfies

$$
\left(R_{n / m} g^{*}\right)(t) \leq \frac{n}{m} g^{*}(t)
$$

and

$$
\begin{aligned}
\left(R_{n / m} g^{*}\right)(t / 2) & \geq(t / 2)^{m / n} \int_{t / 2}^{t} g^{*}(s) s^{-m / n-1} d s \\
& \geq \frac{n}{m}\left[1-2^{-m / n}\right] g^{*}(t), \quad g \in \mathfrak{M}_{+}\left(I_{\Omega}\right), t \in I_{\Omega} .
\end{aligned}
$$


We conclude from the foregoing and (3.4) that

$$
\begin{aligned}
\sigma_{\varrho}(f) & \approx \sigma_{\varrho}\left(t^{-1} \int_{0}^{t} f^{* *}(s) d s\right) \\
& \approx \sup _{\nu(g) \leq 1} \int_{0}^{|\Omega|} t^{-m / n}\left[f^{* *}(t)-f^{*}(t)\right]\left(R_{n / m} g^{*}\right)(t) d t+\int_{0}^{|\Omega|} f^{*}(t) d t \\
& \approx \sup _{\nu\left(R_{n / m} g^{*}\right) \leq 1} \int_{0}^{|\Omega|} t^{-m / n}\left[f^{* *}(t)-f^{*}(t)\right]\left(R_{n / m} g^{*}\right)(t) d t+\int_{0}^{|\Omega|} f^{*}(t) d t \\
& \approx \sup _{\nu(g) \leq 1} \int_{0}^{|\Omega|} t^{-m / n}\left[f^{* *}(t)-f^{*}(t)\right] g^{*}(t) d t+\int_{0}^{|\Omega|} f^{*}(t) d t \\
& \approx \sup _{\bar{\varrho}^{\prime}\left(S_{n / m} g\right) \leq 1} \int_{0}^{|\Omega|} t^{-m / n}\left[f^{* *}(t)-f^{*}(t)\right] g^{*}(t) d t+\int_{0}^{|\Omega|} f^{*}(t) d t,
\end{aligned}
$$

with $f \in \mathfrak{M}_{+}(\Omega), g \in \mathfrak{M}_{+}\left(I_{\Omega}\right)$, as required.

Our next result is a part of Theorem A which seems to be of independent interest.

Theorem 3.3. Let $m, n, \Omega, \varrho$ and $\varrho$ be as in Theorem A. Then, (1.3) implies (1.4).

Proof. As a consequence of Proposition C and (1.3) we have, for $f \in$ $\mathfrak{M}_{+}\left(I_{\Omega}\right)$,

$$
\begin{aligned}
\sigma_{\varrho}(f) & \approx \sup _{\bar{\varrho}^{\prime}(g) \leq 1} \int_{0}^{|\Omega|} t^{-m / n}\left[f^{* *}(t)-f^{*}(t)\right] g^{*}(t) d t+\int_{0}^{|\Omega|} f^{*}(t) d t \\
& \approx\left(\bar{\varrho}^{\prime}\right)_{d}^{\prime}\left(t^{-m / n}\left[f^{* *}(t)-f^{*}(t)\right]\right)+\int_{0}^{|\Omega|} f^{*}(t) d t \\
& \approx \bar{\varrho}\left(\left(s^{-m / n}\left[f^{* *}(s)-f^{*}(s)\right]\right)^{\circ}(t)\right)+\int_{0}^{|\Omega|} f^{*}(t) d t,
\end{aligned}
$$

by (2.6) and the Principle of Duality.

The definition of the level function ensures

$$
\int_{0}^{t} s^{-m / n}\left[f^{* *}(s)-f^{*}(s)\right] d s \leq \int_{0}^{t}\left(y^{-m / n}\left[f^{* *}(y)-f^{*}(y)\right]\right)^{\circ}(s) d s,
$$


from which (2.4) yields

$$
\int_{0}^{t} s^{-m / n}\left[f^{* *}(s)-f^{*}(s)\right] g^{* *}(s) d s \leq \int_{0}^{t}\left(y^{-m / n}\left[f^{* *}(y)-f^{*}(y)\right]\right)^{\circ}(s) g^{* *}(s) d s,
$$

or

$$
\begin{aligned}
\int_{0}^{t} g^{*}(s) \int_{s}^{t} y^{-m / n}\left[f^{* *}(y)\right. & \left.-f^{*}(y)\right] \frac{d y}{y} d s \\
& \leq \int_{0}^{t} g^{*}(s) \int_{s}^{t}\left(z^{-m / n}\left[f^{* *}(z)-f^{*}(z)\right]\right)^{\circ}(y) \frac{d y}{y} d s
\end{aligned}
$$

for $f \in \mathfrak{M}_{+}(\Omega), g \in \mathfrak{M}_{+}\left(I_{\Omega}\right), t \in I_{\Omega}$. But, for $f \in \mathfrak{M}_{+}(\Omega)$,

$$
\begin{aligned}
\int_{t}^{|\Omega|} s^{-m / n} f^{* *} & (s) \frac{d s}{s}=\int_{t}^{|\Omega|} s^{-m / n-2} \int_{0}^{s} f^{*}(y) d y d s \\
= & \int_{0}^{|\Omega|} f^{*}(y) \int_{t}^{|\Omega|} s^{-m / n-2} \chi_{(0, s)}(y) d s d y \\
= & \int_{0}^{t} f^{*}(y) d y \int_{t}^{|\Omega|} s^{-m / n-2} d s+\int_{t}^{|\Omega|} f^{*}(y) \int_{y}^{|\Omega|} s^{-m / n-2} d s d y \\
= & \int_{t}^{|\Omega|} f^{*}(y) \int_{y}^{|\Omega|} s^{-m / n-2} d s d y+\frac{n}{n+m} t^{-n / m} t^{-1} \int_{0}^{t} f^{*}(s) d s \\
& -\frac{n}{n+m}|\Omega|^{-m / n-1} \int_{0}^{t} f^{*}(s) d s, \quad f \in \mathfrak{M}_{+}\left(I_{\Omega}\right),
\end{aligned}
$$

and

$$
\begin{aligned}
\int_{t}^{|\Omega|} f^{*}(y) \int_{y}^{|\Omega|} s^{-m / n-2} d s d y-\int_{t}^{|\Omega|} y^{-m / n-1} f^{*}(y) d y \\
=\frac{n}{n+m} \int_{t}^{|\Omega|} y^{-m / n-1} f^{*}(y) d s-\frac{n}{n+m}|\Omega|^{-m / n-1} \int_{t}^{|\Omega|} f^{*}(y) d y \\
\quad-\int_{t}^{|\Omega|} y^{-m / n-1} f^{*}(y) d y \\
=-\frac{m}{n+m} \int_{t}^{|\Omega|} y^{-m / n-1} f^{*}(y) d y-\frac{n}{n+m}|\Omega|^{-m / n-1} \int_{t}^{|\Omega|} f^{*}(y) d y
\end{aligned}
$$




$$
\geq-\frac{n}{n+m} t^{-m / n} f^{*}(t)-\frac{n}{n+m}|\Omega|^{-m / n-1} \int_{0}^{|\Omega|} f^{*}(t) d t, \quad f \in \mathfrak{M}_{+}\left(I_{\Omega}\right)
$$

Thus,

$$
\begin{aligned}
& \int_{t}^{|\Omega|} s^{-m / n}\left[f^{* *}(s)-f^{*}(s)\right] \frac{d s}{s} \\
& \quad \geq \frac{n}{n+m}\left[f^{* *}(t)-f^{*}(t)\right]-\frac{2 n}{n+m}|\Omega|^{-m / n-1} \int_{0}^{|\Omega|} f^{*}(t) d t,
\end{aligned}
$$

so

$$
\begin{aligned}
& \frac{n}{n+m} \bar{\varrho}\left(t^{-m / n}\left[f^{* *}(t)-f^{*}(t)\right]\right) \\
& \leq \bar{\varrho}\left(\int_{t}^{|\Omega|} s^{-m / n}\left[f^{* *}(s)-f^{*}(s)\right] \frac{d s}{s}\right)+\frac{2 n}{n+m}|\Omega| \bar{\varrho}\left(\chi_{I_{\Omega}}\right) \int_{0}^{|\Omega|} f^{*}(s) d s \\
& \quad \lesssim \bar{\varrho}\left(\int_{t}^{|\Omega|}\left(y^{-m / n}\left[f^{* *}(y)-f^{*}(y)\right]\right)^{\circ}(s) \frac{d s}{s}\right)+\int_{0}^{|\Omega|} f^{*}(t) d t \quad \text { by }(3.8) \text { and HLP } \\
& \quad \lesssim \bar{\varrho}\left(\left(s^{-m / n}\left[f^{* *}(s)-f^{*}(s)\right]\right)^{\circ}(t)\right)+\int_{0}^{|\Omega|} f^{*}(t) d t \\
& \lesssim \sigma_{\varrho}(f), \quad f \in \mathfrak{M}_{+}(\Omega), \quad \text { by }(3.7) ;
\end{aligned}
$$

here we have used the facts that the operator

$$
(Q f)(t):=\int_{t}^{|\Omega|} f(s) \frac{d s}{s}, \quad f \in \mathfrak{M}_{+}\left(I_{\Omega}\right), t \in I_{\Omega}
$$

satisfies

$$
Q: L_{\bar{\varrho}}\left(I_{\Omega}\right) \rightarrow L_{\bar{\varrho}}\left(I_{\Omega}\right) \quad \text { if and only if } \quad P: L_{\bar{\varrho}^{\prime}}\left(I_{\Omega}\right) \rightarrow L_{\bar{\varrho}^{\prime}}\left(I_{\Omega}\right),
$$

and that

$$
L_{\bar{\varrho}^{\prime}}\left(I_{\Omega}\right) \in \operatorname{Int}\left(L_{n /(n-m), \infty}\left(I_{\Omega}\right), L_{\infty}\left(I_{\Omega}\right)\right) .
$$

Since one always has

$$
\sigma_{\varrho}(f) \lesssim \bar{\varrho}\left(t^{-m / n}\left[f^{* *}(t)-f^{*}(t)\right]\right)+\int_{0}^{|\Omega|} f^{*}(t) d t, \quad f \in \mathfrak{M}_{+}(\Omega),
$$

because of $(3.7)$ and $\bar{\varrho}(h) \geq \varrho\left(h^{\circ}\right)$ (by (2.7) and the HLP Principle), the proof is complete.

Corollary 3.4. Let $m, n, \Omega, \varrho$ and $\bar{\varrho}$ be as in Theorem A. Set

$$
\tau(g):=\bar{\varrho}^{\prime}\left(S_{n / m} g^{* *}\right), \quad g \in \mathfrak{M}_{+}(\Omega) .
$$


Then, $\tau$ is an r.i. norm on $\mathfrak{M}_{+}(\Omega)$ and

$$
\sigma_{\varrho}(f) \approx \tau^{\prime}\left(t^{-m / n}\left[f^{* *}(t)-f^{*}(t)\right]\right)+\int_{0}^{|\Omega|} f^{*}(t) d t, \quad f \in \mathfrak{M}_{+}(\Omega) .
$$

Proof. The functional $\tau$ is readily seen to be an r.i. norm such that $L_{\varrho}(\Omega) \subset L_{\tau^{\prime}}(\Omega)$. Moreover, by (1.3),

$$
\bar{\tau}\left(S_{n / m} h\right) \approx \bar{\varrho}^{\prime}\left(S_{n / m}\left(S_{n / m} h\right)\right)=\bar{\varrho}^{\prime}\left(S_{n / m} h\right) \approx \bar{\tau}(h), \quad h \in \mathfrak{M}_{+}\left(I_{\Omega}\right) .
$$

Thus, Theorem 3.3 guarantees

$$
\sigma_{\tau^{\prime}}(f) \approx \tau^{\prime}\left(t^{-m / n}\left[f^{* *}(t)-f^{*}(t)\right]\right)+\int_{0}^{|\Omega|} f^{*}(t) d t, \quad f \in \mathfrak{M}_{+}\left(I_{\Omega}\right) .
$$

But, from Proposition C,

$$
\begin{aligned}
\sigma_{\tau^{\prime}}(f) & =\sup _{\bar{\tau}\left(S_{n / m} g\right) \leq 1} \int_{0}^{|\Omega|} t^{-m / n}\left[f^{* *}(t)-f^{*}(t)\right] g^{*}(t) d t+\int_{0}^{|\Omega|} f^{*}(t) d t \\
& \approx \sup _{\bar{\tau}(g) \leq 1} \int_{0}^{|\Omega|} t^{-m / n}\left[f^{* *}(t)-f^{*}(t)\right] g^{*}(t) d t+\int_{0}^{|\Omega|} f^{*}(t) d t \quad \text { by }(3.11) \\
& \approx \sup _{\varrho^{\prime}\left(S_{n / m} g\right) \leq 1} \int_{0}^{|\Omega|} t^{-m / n}\left[f^{* *}(t)-f^{*}(t)\right] g^{*}(t) d t+\int_{0}^{|\Omega|} f^{*}(t) d t \\
& \approx \sigma_{\varrho}(f), \quad f \in \mathfrak{M}_{+}(\Omega),
\end{aligned}
$$

and (3.10) follows.

REMARK 3.5. Some r.i. norms $\mu$ require $h^{*}$ in order to compute $\mu(h)$. Should this prove difficult for the $\mu=\bar{\varrho}$ and $h(t)=t^{-m / n}\left[f^{* *}(t)-f^{*}(t)\right]$ in (1.4), the first paragraph of the proof of Theorem 3.3, together with (A.1) below, offers an alternative expression, given $P: L_{\bar{\varrho}}(\Omega) \rightarrow L_{\bar{\varrho}}(\Omega)$, namely,

$$
\sigma_{\varrho}(f) \approx \bar{\varrho}\left(\sup _{t \leq s<|\Omega|} s^{-1} \int_{0}^{s} y^{-m / n}\left[f^{* *}(y)-f^{*}(y)\right] d y\right)+\int_{0}^{|\Omega|} f^{*}(t) d t,
$$

for $f \in \mathfrak{M}(\Omega)$. Here, the function $h$ to which the norm $\bar{\varrho}$ is applied is its own rearrangement.

\section{Proofs of Theorems A and B}

Proof of Theorem A. By [4, Corollary 3.14],

$$
L_{\bar{\varrho}}\left(I_{\Omega}\right) \in \operatorname{Int}\left(L_{1}\left(I_{\Omega}\right), L_{n / m, 1}\left(I_{\Omega}\right)\right) \text {. }
$$


Theorem 2.2 then yields

$$
S_{n / m}: L_{\overline{\varrho_{\sigma}}}\left(I_{\Omega}\right) \rightarrow L_{\overline{\varrho_{\sigma}}}\left(I_{\Omega}\right),
$$

and this, by Theorem 3.3, implies

$$
\sigma_{\varrho_{\sigma}}(f) \approx \overline{\varrho_{\sigma}}\left(t^{-m / n}\left[f^{* *}(t)-f^{*}(t)\right]\right)+\int_{0}^{|\Omega|} f^{*}(t) d t, \quad f \in \mathfrak{M}_{+}(\Omega) .
$$

Further, Proposition 5.2 in [4] guarantees

$$
\varrho_{\sigma_{\varrho}}(f) \approx \overline{\sigma_{\varrho}}\left(\int_{t}^{|\Omega|} f^{*}(s) s^{m / n-1} d s\right), \quad f \in \mathfrak{M}_{+}(\Omega) .
$$

When $\varrho$ is optimal in (1.1), $\varrho \approx \varrho_{\sigma}$, so (1.3) holds, by (4.1), and (4.2) becomes (1.4).

Given (1.3), we have (1.4), in view of Corollary 3.4. We claim that (4.3) and (1.4) together ensure

$$
\varrho_{\sigma \varrho}(f) \approx \varrho(f), \quad f \in \mathfrak{M}_{+}(\Omega),
$$

and, hence, the optimality of $\varrho$ in (1.1). Indeed, for $f \in \mathfrak{M}_{+}(\Omega)$,

$$
\begin{aligned}
\varrho_{\sigma_{\varrho}}(f) \approx & \overline{\sigma_{\varrho}}\left(\int_{t}^{|\Omega|} f^{*}(s) s^{m / n-1} d s\right) \\
\approx & \bar{\varrho}\left(t^{-m / n}\left[t^{-1} \int_{0}^{t|\Omega|} \int_{s} f^{*}(y) y^{m / n-1} d y d s-\int_{t}^{|\Omega|} f^{*}(s) s^{m / n-1} d s\right]\right) \\
& +\int_{0}^{|\Omega||\Omega|} \int_{t}^{|\Omega|} f^{*}(s) s^{m / n-1} d s d t \quad \text { by }(1.4) \\
\approx & \bar{\varrho}\left(t^{-m / n-1} \int_{0}^{t} f^{*}(s) s^{m / n} d s\right),
\end{aligned}
$$

since

$$
t^{-1} \int_{0}^{t|\Omega|} \int_{s} f^{*}(y) y^{m / n-1} d y d s=t^{-1} \int_{0}^{t} f^{*}(s) s^{m / n} d s+\int_{t}^{|\Omega|} f^{*}(s) s^{m / n-1} d s
$$

and

$$
\begin{aligned}
\int_{0}^{|\Omega| \mid} \int_{t}^{|\Omega|} f^{*}(s) s^{m / n-1} d s d t & =\int_{0}^{|\Omega|} f^{*}(s) s^{m / n} d s \\
& =C \bar{\varrho}\left(\int_{0}^{|\Omega|} f^{*}(s) s^{m / n} d s\right) \quad\left(C=\bar{\varrho}\left(\chi_{I_{\Omega}}\right)^{-1}\right)
\end{aligned}
$$




$$
\leq C \bar{\varrho}\left(\int_{0}^{|\Omega|} f^{*}\left(\frac{t s}{|\Omega|}\right) s^{m / n} d s\right) \leq C|\Omega|^{m / n-1} \bar{\varrho}\left(t^{-m / n-1} \int_{0}^{t} f^{*}(s) s^{m / n} d s\right) .
$$

The operator

$$
f \mapsto t^{-m / n-1} \int_{0}^{t} f(s) s^{m / n} d s
$$

is the associate of the operator $R_{n / m}$ in the proof of Proposition $\mathrm{C}$, and therefore

$$
\bar{\varrho}\left(t^{-m / n-1} \int_{0}^{t} f^{*}(s) s^{m / n} d s\right) \lesssim \bar{\varrho}(f), \quad f \in \mathfrak{M}_{+}(\Omega) .
$$

But

$$
t^{-m / n-1} \int_{0}^{t} f^{*}(s) s^{m / n} d s \geq \frac{n}{n+m} f^{*}(t), \quad t \in I_{\Omega},
$$

whence

$$
\bar{\varrho}(f) \approx \bar{\varrho}\left(t^{-m / n-1} \int_{0}^{t} f^{*}(s) s^{m / n} d s\right) \approx \varrho_{\sigma_{\varrho}}(f), \quad f \in \mathfrak{M}_{+}(\Omega) .
$$

The proof of the assertion concerning the optimality of $\sigma$ is similar to the one for $\varrho$. Thus, if $\sigma$ is optimal in (1.1), then $\sigma \approx \sigma_{\varrho}$ and (1.5) holds by [4, Theorem 3.12]; in that case, (1.7) is satisfied.

Given (1.5), Proposition 5.2 in [4] ensures (1.6). Using (4.2) and (1.6), we will obtain

$$
\sigma_{\varrho_{\sigma}}(f) \approx \sigma(f), \quad f \in \mathfrak{M}_{+}(\Omega),
$$

and thus, the optimality of $\sigma$ in (1.1). In fact, it suffices to show

$$
\sigma_{\varrho_{\sigma}}(f) \lesssim \sigma(f), \quad f \in \mathfrak{M}_{+}(\Omega) .
$$

Now, if $0<t<|\Omega| / 2$, then

$$
\begin{aligned}
\int_{t}^{|\Omega|} s^{-m / n} & {\left[f^{* *}(s)-f^{*}(s)\right] \frac{d s}{s} } \\
= & \int_{t}^{|\Omega|} s^{-m / n-2} \int_{0}^{s} f^{*}(y) d y d s-\int_{t}^{|\Omega|} s^{-m / n-1} f^{*}(s) d s \\
= & \int_{t}^{|\Omega|} s^{-m / n-2} d s \int_{0}^{t} f^{*}(y) d y+\int_{t}^{|\Omega|} s^{-m / n-2} \int_{t}^{s} f^{*}(y) d y d s \\
& -\int_{t}^{|\Omega|} s^{-m / n-1} f^{*}(s) d s
\end{aligned}
$$




$$
\begin{aligned}
\geq & \int_{t}^{|\Omega|} s^{-m / n-2} d s \int_{0}^{t} f^{*}(y) d y+\int_{t}^{|\Omega|} s^{-m / n-2}(s-t) f^{*}(s) d s \\
& \quad-\int_{t}^{|\Omega|} s^{-m / n-1} f^{*}(s) d s \\
= & \int_{t}^{|\Omega|} s^{-m / n-2} d s \int_{0}^{t} f^{*}(y) d y-t \int_{t}^{|\Omega|} s^{-m / n-2} f^{*}(s) d s \\
\geq & t \int_{t}^{2 t} s^{-m / n-2} d s\left[f^{* *}(t)-f^{*}(t)\right] \\
\geq & \frac{1}{2} \frac{n}{n+m} t^{-m / n}\left[f^{* *}(t)-f^{*}(t)\right],
\end{aligned}
$$

while if $|\Omega| / 2 \leq t<|\Omega|$, then

$$
t^{-m / n}\left[f^{* *}(t)-f^{*}(t)\right] \leq\left(\frac{2}{|\Omega|}\right)^{m / n+1} \int_{0}^{|\Omega|} f^{*}(t) d t, \quad f \in \mathfrak{M}_{+}(\Omega) .
$$

We conclude that when $f \in \mathfrak{M}_{+}(\Omega)$,

$$
\begin{aligned}
& \sigma_{\varrho_{\sigma}}(f) \approx \overline{\varrho_{\sigma}}\left(t^{-m / n}\left[f^{* *}(t)-f^{*}(t)\right]\right)+\int_{0}^{|\Omega|} f^{*}(t) d t \quad \text { by }(4.2) \\
& \lesssim \overline{\varrho_{\sigma}}\left(\int_{t}^{|\Omega|} s^{-m / n}\left[f^{* *}(s)-f^{*}(s)\right] \frac{d s}{s}\right)+\int_{0}^{|\Omega|} f^{*}(t) d t \\
& \lesssim \bar{\sigma}\left(\int_{t}^{|\Omega|}\left[\int_{s}^{|\Omega|} y^{-m / n}\left[f^{* *}(y)-f^{*}(y)\right] \frac{d y}{y}\right] s^{m / n-1} d s\right) \\
& |\Omega| \\
& +\int_{0} f^{*}(t) d t \quad \text { by }(4.3) \\
& \lesssim \bar{\sigma}\left(\int_{t}^{|\Omega|} s^{-m / n}\left[f^{* *}(s)-f^{*}(s)\right] s^{m / n-1} d s\right)+\int_{0}^{|\Omega|} f^{*}(t) d t \\
& =\bar{\sigma}\left(\int_{t}^{|\Omega|} s^{-1} \int_{0}^{s} f^{*}(y) d y \frac{d s}{s}-\int_{t}^{|\Omega|} f^{*}(s) \frac{d s}{s}\right)+\int_{0}^{|\Omega|} f^{*}(t) d t \\
& \lesssim \bar{\sigma}\left(t^{-1} \int_{0}^{t} f^{*}(s) d s-\frac{1}{|\Omega|} \int_{t}^{\mid \Omega} f^{*}(s) d s\right)+\int_{0}^{|\Omega|} f^{*}(t) d t \\
& \lesssim \bar{\sigma}\left(t^{-1} \int_{0}^{t} f^{*}(s) d s\right)+\int_{0}^{|\Omega|} f^{*}(t) d t \lesssim \bar{\sigma}\left(t^{-1} \int_{0}^{t} f^{*}(s) d s\right),
\end{aligned}
$$


since

$$
\begin{aligned}
\int_{t}^{|\Omega| \mid} \int_{s}^{|\Omega|} h(y) \frac{d y}{y} s^{m / n-1} d s & =\int_{t}^{|\Omega|} \frac{h(y)}{y} \int_{t}^{y} s^{m / n-1} d s d y \\
& =\frac{n}{m} \int_{t}^{|\Omega|} \frac{h(y)}{y}\left[y^{m / n}-t^{m / n}\right] d y \\
& \leq \frac{n}{m} \int_{t}^{|\Omega|} h(y) y^{m / n-1} d y
\end{aligned}
$$

and

$$
\begin{aligned}
& \int_{t}^{|\Omega|} s^{-1} \int_{0}^{s} h(y) d y \frac{d s}{s}=\int_{t}^{|\Omega|} s^{-2} d s \int_{0}^{t} h(y) d y+\int_{t}^{|\Omega|} s^{-2} \int_{t}^{s} h(y) d y d s \\
& \quad \leq t^{-1} \int_{0}^{t} h(y) d y+\int_{t}^{|\Omega|} h(y) \frac{d y}{y}-\frac{1}{|\Omega|} \int_{t}^{|\Omega|} h(y) d y, \quad h \in \mathfrak{M}_{+}\left(I_{\Omega}\right) .
\end{aligned}
$$

Finally, (1.5) and [4, Theorem 3.12] imply, as in the proof of Proposition C, that

$$
P: L_{\bar{\sigma}}\left(I_{\Omega}\right) \rightarrow L_{\bar{\sigma}}\left(I_{\Omega}\right),
$$

which means

$$
\bar{\sigma}\left(t^{-1} \int_{0}^{t} f^{*}(s) d s\right) \approx \bar{\sigma}(f), \quad f \in \mathfrak{M}_{+}(\Omega) .
$$

Proof of Theorem B. We know the following:

$$
\begin{aligned}
& \varrho_{D} \lesssim \varrho, \quad \text { or equivalently, } \quad \varrho^{\prime} \lesssim \varrho_{D}^{\prime} ; \\
& \varrho^{\prime} \lesssim \mu, \quad \text { or equivalently, } \mu^{\prime} \lesssim \varrho ; \\
& S_{n / m}: L_{\bar{\varrho}_{D}^{\prime}}\left(I_{\Omega}\right) \rightarrow L_{\bar{\varrho}_{D}^{\prime}}\left(I_{\Omega}\right) ; \\
& S_{n / m}: L_{\bar{\mu}^{\prime}}\left(I_{\Omega}\right) \rightarrow L_{\bar{\mu}^{\prime}}\left(I_{\Omega}\right) .
\end{aligned}
$$

Now, (4.4) and (4.6) yield

$$
\mu(g)=\bar{\varrho}^{\prime}\left(S_{n / m} g^{* *}\right) \approx \bar{\varrho}^{\prime}\left(S_{n / m} g^{*}\right) \lesssim \bar{\varrho}_{D}^{\prime}\left(S_{n / m} g^{*}\right) \lesssim \bar{\varrho}_{D}^{\prime}\left(g^{*}\right), \quad g \in \mathfrak{M}_{+}(\Omega),
$$

and, hence, $\varrho_{D} \lesssim \mu^{\prime}$. So, keeping (4.5) in mind, we see that

$$
\varrho_{D} \lesssim \mu^{\prime} \lesssim \varrho
$$

Since $\sigma_{\varrho_{D}}=\sigma_{\varrho}$, we conclude $\sigma_{\mu^{\prime}}=\sigma_{\varrho}$, that is,

$$
W^{m, \mu^{\prime}}(\Omega) \hookrightarrow L_{\sigma_{\varrho}}(\Omega),
$$

which, in view of (4.7) and Theorem A, means $\mu^{\prime} \approx \varrho_{D}$. 
5. Examples. We here illustrate Theorem A in the context of Orlicz spaces.

An Orlicz norm is defined in terms of a Young function $A(t)=\int_{0}^{t} a(s) d s$, with $a(s)$ increasing on $\mathbb{R}_{+}, a(0+)=0$ and $\lim _{s \rightarrow \infty} a(s)=\infty$. Given a domain $\Omega \subset \mathbb{R}^{n}$, the (Luxemburg) Orlicz (r.i.) norm, $\varrho_{A}$, is defined at $f \in$ $\mathfrak{M}_{+}\left(I_{\Omega}\right)$ by

$$
\varrho_{A}(f)=\inf \left\{\lambda>0: \int_{I_{\Omega}} A\left(\frac{f(t)}{\lambda}\right) d t=\int_{I_{\Omega}} A\left(\frac{f^{*}(t)}{\lambda}\right) d t \leq 1\right\}
$$

and at $f \in \mathfrak{M}_{+}(\Omega)$ by

$$
\varrho_{A}(f)=\inf \left\{\lambda>0: \int_{I_{\Omega}} A\left(\frac{f^{*}(t)}{\lambda}\right) d t \leq 1\right\} .
$$

The Köthe norm dual to $\varrho_{A}$ is equivalent to the Orlicz norm $\varrho_{\widetilde{A}}$, where

$$
\widetilde{A}(t):=\int_{0}^{t} a^{-1}(s) d s, \quad t>0,
$$

is the Young function complementary to $A$; in fact,

$$
\varrho_{\widetilde{A}}(g) \leq \varrho_{A}^{\prime}(g) \leq 2 \varrho_{\widetilde{A}}(g), \quad g \in \mathfrak{M}_{+}\left(I_{\Omega}\right) .
$$

In [3] we determined precisely when $S_{n / m}$ and $T_{n / m}$ are bounded between Orlicz spaces. Theorems B and 5.2 of that paper yield, respectively, Theorems 5.1 and 5.2 below.

Theorem 5.1. Let $m, n$ and $\Omega$ be as in Theorem $A$ and suppose $A$ is a Young function whose complementary function, $\widetilde{A}$, satisfies

$$
\widetilde{A}(t)=0, t \in I_{\Omega}, \quad \text { and } \quad L_{\varrho_{\widetilde{A}}}(\Omega) \subsetneq L_{n /(n-m), \infty}(\Omega) .
$$

Then $\varrho=\varrho_{A}$ is optimal in (1.1) for some r.i. norm $\sigma$ on $\mathfrak{M}(\Omega)$ if and only if

$$
\int_{|\Omega|}^{t} \frac{\widetilde{A}(s)}{s^{n /(n-m)+1}} d s \leq \frac{\widetilde{A}(K t)}{t^{n /(n-m)}}, \quad t \gg|\Omega| .
$$

Moreover, in that case,

$$
\sigma_{\varrho_{A}}(f) \approx \varrho_{A}\left(t^{-m / n}\left[f^{* *}(t)-f^{*}(t)\right]\right)+\int_{0}^{|\Omega|} f^{*}(t) d t, \quad f \in \mathfrak{M}_{+}(\Omega) .
$$

TheOREm 5.2. Let $m, n$ and $\Omega$ be as in Theorem $A$ and suppose $A$ is a Young function whose complementary function, $\widetilde{A}$, satisfies

$$
\widetilde{A}(t)=0, t \in I_{\Omega}, \quad \text { and } \quad L_{n / m, \infty}(\Omega) \subsetneq L_{\varrho_{\widetilde{A}}}(\Omega) .
$$


Then $\sigma=\varrho_{A}$ is optimal in (1.1) for some r.i. norm $\varrho$ on $\mathfrak{M}_{+}(\Omega)$ if and only if

$$
\int_{t}^{\infty} \frac{\widetilde{A}(s)}{s^{n / m+1}} d s \leq \frac{\widetilde{A}(K t)}{t^{n / m}}, \quad t \gg|\Omega| .
$$

Moreover, in that case,

$$
\varrho_{\sigma}(f) \approx \varrho_{A}\left(\int_{t}^{|\Omega|} f^{*}(s) s^{m / n-1} d s\right), \quad f \in \mathfrak{M}_{+}(\Omega) .
$$

Appendix. The following result concerning the level function, $f^{\circ}$, of an $f \in \mathfrak{M}_{+}\left(I_{\Omega}\right)$, was communicated to us by G. Sinnamon.

Theorem A.1. For any $f \in \mathfrak{M}_{+}\left(I_{\Omega}\right)$, the function

$$
q(t):=t \sup _{t \leq s<1} s^{-1} \int_{0}^{s} f(y) d y
$$

is quasiconcave on $I_{\Omega}$. Moreover,

$$
q(t) \leq \int_{0}^{t} f^{\circ}(s) d s \leq 2 q(t) .
$$

Proof (A. Gogatishvili). Set $f(s)=0$ for $s>1$ so that

$$
q(t)=t \sup _{t \leq s<\infty} s^{-1} \int_{0}^{s} f(y) d y, \quad t \in I_{\Omega} .
$$

Since $q(t) / t$ is clearly nonincreasing, we need only verify that $q(t)$ is nondecreasing to get $q$ quasiconcave on $I_{\Omega}$. But this is readily seen from

$$
t \sup _{t \leq s<\infty} s^{-1} \int_{0}^{s} f(y) d y=\sup _{1 \leq s<\infty} s^{-1} \int_{0}^{t s} f(y) d y .
$$

As $q(t) \geq \int_{0}^{t} f(y) d y$, the least concave majorant of $q$ dominates $\int_{0}^{t} f(y) d y$ and hence $\int_{0}^{t} f^{\circ}(s) d s$. The least concave majorant of a quasiconcave function $q(t)$ being no greater than $2 q(t)$, we have the second of the inequalities in (A.1).

Observe that

$$
\int_{0}^{t} f^{\circ}(s) d s=\sup _{t_{1} \leq t, 0<t_{2}<\infty} \frac{t_{2} \int_{0}^{t-t_{1}} f(s) d s+t_{1} \int_{0}^{t+t_{2}} f(s) d s}{t_{1}+t_{2}}, \quad 0<t<1 .
$$

Fix $s$ and $t$ with $t \leq s<1$. Set $t_{1}=t$ and $t_{2}=s-t$. Then

$$
\frac{t_{2} \int_{0}^{t-t_{1}} f(s) d s+t_{1} \int_{0}^{t+t_{2}} f(s) d s}{t_{1}+t_{2}}=\frac{t}{s} \int_{0}^{s} f(y) d y
$$


whence

$$
q(t) \leq \int_{0}^{t} f^{\circ}(s) d s
$$

and we are done.

Acknowledgments. We would like to thank the referee for her/his critical reading of the paper and many valuable comments.

This research was supported in part by NSERC grant A4021, grant MSM 0021620839 of the Czech Ministry of Education, grants 201/01/0333, 201/03/0935, 201/05/2033 and 201/08/0383 of the Grant Agency of the Czech Republic, NATO grant PST.CLG.978798 and Leverhulme Trust Grant no. $\mathrm{F} / 00407 / \mathrm{E}$.

\section{References}

[1] C. Bennett and R. Sharpley, Interpolation of Operators, Pure Appl. Math. 129, Academic Press, Boston, 1988.

[2] D. E. Edmunds, R. Kerman and L. Pick, Optimal Sobolev imbeddings involving rearrangement-invariant quasinorms, J. Funct. Anal. 170 (2000), 307-355.

[3] R. Kerman, C. Phipps and L. Pick, Boundedness criteria for certain supremum operators on Lorentz and Orlicz spaces, in preparation.

[4] R. Kerman and L. Pick, Optimal Sobolev imbeddings, Forum Math. 18 (2006), 535570 .

[5] - - - Compactness of Sobolev imbeddings involving rearrangement-invariant norms, Studia Math. 186 (2008), 127-160.

[6] J. Lindenstrauss and L. Tzafriri, Classical Banach Spaces I and II, Springer, Berlin, 1996.

[7] G. Sinnamon, The level function in rearrangement-invariant spaces, Publ. Mat. 45 (2001), 175-198.

[8] -, personal communication, 2001.

Department of Mathematics

Brock University

500 Glenridge Avenue

St. Catharines

Ontario, Canada L2S 3A1

E-mail: rkerman@brocku.ca
Department of Mathematical Analysis Faculty of Mathematics and Physics Charles University Sokolovská 83 18675 Praha 8, Czech Republic E-mail: pick@karlin.mff.cuni.cz

Received October 3, 2006

Revised version January 26, 2009 